\title{
Clean measurements of the nucleon axial-vector and free-neutron magnetic form factors
}

\author{
A. Deur \\ Thomas Jefferson National Accelerator Facility, Newport News, VA 23606
}

(Dated: October 1, 2018)

\begin{abstract}
We discuss the feasibility of a weak charged current experiment using a low energy electron beam. A first goal is to measure the $\mathrm{Q}^{2}$ dependence of the axial-vector form factor $g_{a}\left(Q^{2}\right)$. It can be measured model-independently and as robustly as for electromagnetic form factors from typical electron scattering experiments, in contrast to the methods used so far to measure $g_{a}\left(Q^{2}\right)$. If $g_{a}\left(Q^{2}\right)$ follows a dipole form, the axial mass can be extracted with a better accuracy than the world data altogether. The most important detection equipment would be a segmented neutron detector with good momentum and angular resolution that is symmetric about the beam direction, and covers a moderate angular range. A high intensity beam $(100 \mathrm{uA})$ is necessary. Beam polarization is highly desirable as it provides a clean measurement of the backgrounds. Beam energies between 70 and $110 \mathrm{MeV}$ are ideal. This range would provide a $Q^{2}$ mapping of $g_{a}$ between $0.01 ; Q^{2} ; 0.04$ $\mathrm{GeV}^{2}$. 60 days of beam can yield 14 data points with a subpercent statistical and point to point uncorrelated uncertainties on each point. Such an experiment may also allow to measure the freeneutron magnetic form factor $G_{M}^{n}$. The experiment employs the usual techniques of electron-nucleon scattering and presents no special difficulty. Higher energy extensions are possible. They could yield measurements of $g_{a}\left(Q^{2}\right)$ up to $Q^{2}=3 \mathrm{GeV}^{2}$ and the possibility to access other form factors, such as the almost unknown pseudoscalar form factor $g_{P}$. However, the experiments become much more challenging as soon as beam energies pass the pion production threshold.
\end{abstract}

PACS numbers: 114.20.Dh

\section{MOTIVATION}

Form factors are fundamental quantities describing hadrons and provide crucial insight into their structure. Their precise measurements are benchmarks for the theories and phenomenologies aiming at describing the hadronic and nuclear structure, such as Lattice QCD, Chiral Perturbation Theory or QCD counting rules.

Lepton scattering off a nucleon is described by 4 form factors: The electromagnetic form factors $G_{E}^{N}\left(Q^{2}\right)$ and $G_{M}^{N}\left(Q^{2}\right)$ (where $N$ indicates the proton or the neutron) and the axial-vector and induced pseudoscalar form factors $g_{A}\left(Q^{2}\right)$ and $g_{p}\left(Q^{2}\right) . g_{p}$ is almost unknown and is interpreted as arising from scattering off the meson cloud made dominantly of pions. $g_{A}$ is better known than $g_{p}$ but much less than $G_{E}^{N}$ and $G_{M}^{N}$, although it is of the same importance. In particular, precise lattice QCD predictions exist for $g_{A}$, see e.g. [1]. There is no precise and accurate data for $g_{A}$ because it is measured from either neutrino elastic scattering (with both weak charged and neutral currents), or pion electroproduction. Neutrino experiments are delicate to carry out, accumulate statistics slowly, relies on Monte Carlo simulations and are done typically on dense nuclear targets such as iron, rather than free nucleons, although the most recent experiments were carried on lighter targets $\left({ }^{12} \mathrm{C}\right.$ and $\left.{ }^{16} \mathrm{O}\right)$. Pion data need model-dependent corrections to be interpreted. Indeed, neutrino and pion data disagreed until 2002 when new corrections based on baryon chiral perturbation theory seemed to solve the disagreement, see [2]. It is important to independently check these corrections. Furthermore, new tensions between experimental results arose with the most recent neutrino experiments that found a shallower $Q^{2}$-dependence of $g_{A}\left(Q^{2}\right)$. Parametrizing it with a dipole form: $g_{A}\left(Q^{2}\right)=g_{a} /\left(1+Q^{2} / M_{A}^{2}\right)^{2}$, defines the nucleon axial mass $M_{A}$. Earlier experiments measured $M_{A}=1.03 \pm 0.02 \mathrm{GeV}$ while the recent $\mathrm{K} 2 \mathrm{~K}$ experiment [3] yields $M_{A}=1.20 \pm 0.12$ from ${ }^{16} \mathrm{O}$ and $M_{A}=1.14 \pm 0.11$ from ${ }^{12} \mathrm{C}$ and the MiniBooNE collaboration measured $M_{A}=1.35 \pm 0.17$ from ${ }^{12} \mathrm{C}[4]$.

Consequently, it is important to provide a third, more robust, way to measure $g_{A}\left(Q^{2}\right)$. To do so, we propose to measure the weak charged current reaction $e+p \rightarrow \nu+n$ using a $\sim 100 \mathrm{MeV}$ electron beam. Some of the material presented here is from [5], which discusses a similar (but more difficult) experiment with GeV electron beams.

Another motivation for this measurement is to obtain the free-neutron form factors. Neutron structure information is so far extracted from nuclear targets $\left(\mathrm{D},{ }^{3} \mathrm{He}\right)$, which involves nuclear corrections. It is obviously desirable to obtain the information from a free neutron. Related interests in this program, such as measuring $g_{A}\left(Q^{2}\right)$ in ${ }^{3} \mathrm{He}$, investigating second class currents or obtaining the ratio of the axial to vector coupling constants in a novel way, are discussed in [6]. Similar programs had also been discussed in [7]. 


\section{EXPERIMENT}

Although the elastic reaction $e+p \rightarrow \nu+n$ has been considered for a long time, no experiment has yet been done due to several difficulties. One is that there are only neutral particles in the final state. We will not consider detecting the neutrino but only the recoiling neutron. Although detection of neutrons is routinely done, it is difficult to determine their kinematics to a level at which the elastic reaction can be cleanly selected. Furthermore, a weak cross section is typically $10^{-11}$ times smaller than its electromagnetic (EM) counterpart. Consequently, the weak reaction is buried deep under the EM background. One well established solution to this problem is to measure the interference term between the EM and the weak reactions using the resulting small (typically ppm) single spin asymmetry (parity violating experiments, see e.g. the reviews [8]. However, this technique only allows us to study reactions with the same final states as the EM reactions, that is only with the neutral weak current. Another strategy to reduce the background to a similar level as for the PV technique, but allowing to access weak charged current, is to select the backward reaction. There, the undetected neutrinos recoil at large angles and the neutrons are detected at small angles. The Weak/EM cross section ratio is enhanced to about $6 \times 10^{-6}$ for a $100 \mathrm{MeV}$ beam and a lepton scattering angle of $150^{\circ}$ (corresponding to a nucleon recoil angle of $14^{\circ}$. The small backward cross-sections require luminosities of $10^{39}$ to $10^{40} \mathrm{~cm}^{-2} \mathrm{~s}^{-1}$. The beam energy should be below $\sim 150 \mathrm{MeV}$ to avoid the pion production since this can produce a neutron in the final state with a proton in the initial one. The beam must be polarized to reduce the EM background and pulsed to measure the neutron energy with TOF technique and to avoid the prompt EM background (photon flash). We list below the main experimental components:

Beam: We assume a $63 \mathrm{MHz}$ beam structure (already used with the Jefferson Lab CEBAF beam). The average current is assumed to be $100 \mu \mathrm{A}$, leading to a peak current of about $800 \mu \mathrm{A}$. The beam polarization is the main ingredient to cleanly subtract the EM background: The charged current asymmetry is $100 \%$ and the elastic EM one is 0. Thus, helicity minus beam pulses allow for both the weak and EM reactions, while helicity plus pulses allow for the EM reaction only. Then, subtracting events from minus helicity pulses to events from helicity plus pulses cleanly yields the weak reaction. However, the larger the EM background, the longer the experiment needs to be so that the background statistical fluctuations are small compared to the expected precision of the experiment. It is thus necessary to have other means to reduce the EM background before subtracting it from the weak signal.

Sweeping magnet: A sweeping magnet is needed to sweep the protons away from the neutron detector acceptance. At the low energies considered, a simple warm magnet is adequate. It will also sweep away the electrons. Those could be disposed of with the TOF cuts (prompt EM background) but at the large luminosities considered the single electron rate would be too large for the DAQ to handle.

Backward detector: A high detection efficiency backward detector is necessary to veto out EM reactions that produced a neutron. Events with a recoiling electron detected in coincidence with neutron are flagged (there is no need to cut them at trigger level because of the low counting rate. In addition, such data are necessary to extract $g_{p}$ in higher energy versions of the experiment discussed here). We assume a $10^{-3}$ detection inefficiency. It can be reached using existing calorimeters used in JLab experiments such as E1-DVCS [9] or PrimEx [10]. The efficiency can be further improved by adding scintillators between the detector and the target. The detector must cover a $90^{\circ}$ to $170^{\circ}$ azimuthal angle and a $2 \pi$ polar angle to match the solid angle of the neutron detector.

Neutron detector: The neutron detector must be typically $2 \mathrm{~m}$ away from the target to allow TOF measurements compatible with the beam pulsed structure: It allows to separate the photons from the neutrons and leave enough time for all relevant neutrons to reach the detector before the next photon flash. The detector should have a large acceptance to yield reasonable counting rates. We assume a $5^{\circ}$ to $45^{\circ}$ azimuthal angle coverage and a $2 \pi$ polar angle coverage. (Complete polar angle coverage is also important for background management, as we will discuss.) Likewise, the detection must be efficient. The detector should be segmented to permit angular determination. This, associated with the TOF, can allow a selection of the elastic reaction and rejection of EM inelastic backgrounds. Finally, the detector must be shielded against low energy backgrounds and surrounded by scintillator paddles to veto any remnant of charged background (e.g. rescattering of charged particles after being swept, cosmic rays).

Target: The cell must be long for high luminosity and to maximize cell length over window thickness. However, since the reaction vertex cannot be determined, the target length contributes directly to the TOF uncertainty. $20 \mathrm{~cm}$ is a good compromise. Deuterium impurities must be minimized ( $5 \mathrm{ppm}$ is available industrially and is enough).

With this setup, we estimate that the EM backgrounds should be manageable: 10 mil Be cell windows yield a neutron background from $\mathrm{Be}+\mathrm{e}^{-} \rightarrow \mathrm{n}+\mathrm{e}^{-}+\mathrm{X}$ with a noise/signal ratio of $8 \times 10^{3}$ at worst $\left(\mathrm{E}=0.11 \mathrm{GeV}, 45^{\circ}\right)$. It can be reduced to unity with the backward electron detector rejecting the $(\mathrm{e}-\mathrm{n})$ coincidences and decreased further by selecting the nucleon elastic reaction. A 5 ppm level D contamination would yield a noise to signal ratio of about 0.2 , becoming negligible after rejection from the backward detector. The backward detector also reduces to a negligible level the neutron contamination originating from elastically scattered protons undergoing charge exchange while crossing the material surrounding the cell, and from quasi-elastic EM reaction between the Al cell walls and electrons of the beam halo or Moller electrons produced in the cell. 


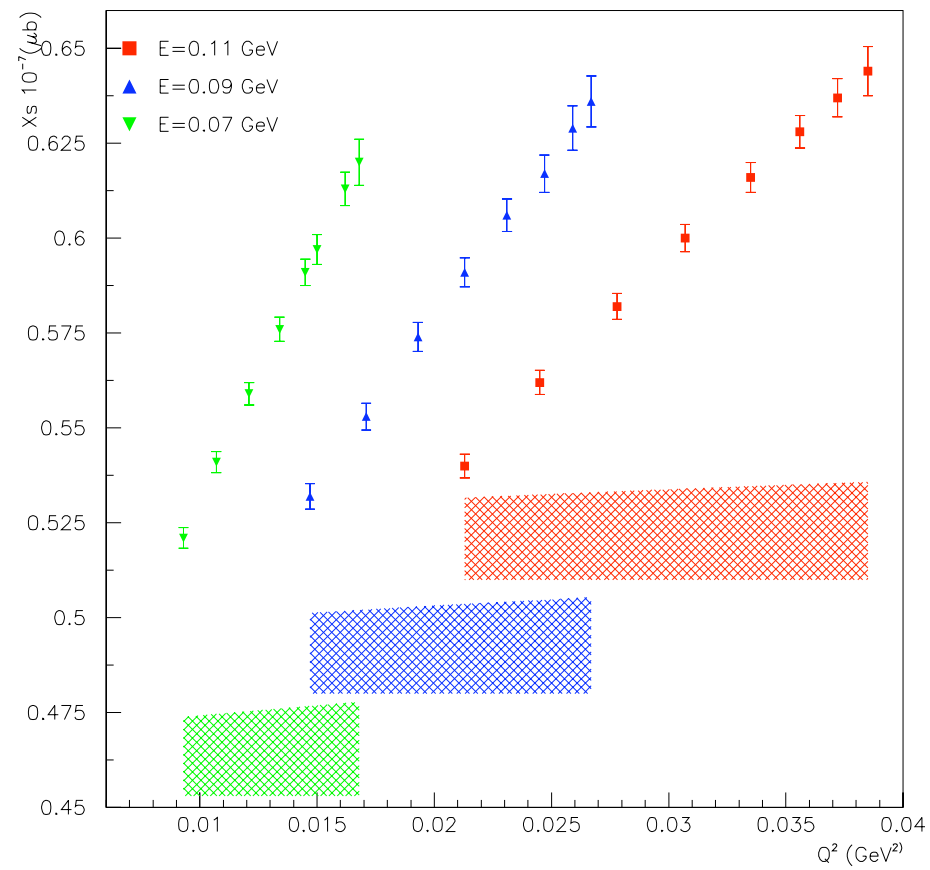

FIG. 1: Expected cross-sections and uncertainties for 6 days of data taking at $110 \mathrm{MeV}, 7$ days at $90 \mathrm{MeV}$ and 17 days at 70 $\mathrm{MeV}$. The error bars represent the statistical uncertainty and the bands the systematic ones. The uncertainties assume a $100 \%$ experimental efficiency and negligible backgrounds.

In Fig. II we show the expected cross-sections with uncertainties using the above experimental setup and for 6 days of running at $110 \mathrm{MeV}, 7$ days at $90 \mathrm{MeV}$ and 17 days at $70 \mathrm{MeV}$. The error bars are statistical only and the bands represent an assumed $4 \%$ systematics. For the statistical errors, we took a $100 \%$ experimental efficiency (neutron detector, beam polarization) and a signal/backgroud $\leq 1$. The typical reaction rates are a few $\%$ of $\mathrm{Hz}$ for the weak reaction and a few $10^{4} \mathrm{~Hz}$ for the EM background. From such data, 14 different $Q^{2}$ points can be extracted with an additional 10 overlapping points. This would provide a $Q^{2}$ mapping of $g_{A}\left(Q^{2}\right)$ with unprecedented precision. One can compare the subpercent statistical uncertainty of a single point to the full statistical precision of $\sim 13 \%$ of the latest neutrino experiment [4].

In principle, at the same $Q^{2}$ but different angles (i.e. beam energies), $g_{A}$ and $G_{M}^{n}$ can be separated. $\left(G_{M}^{p}, G_{E}^{p}\right.$ and $G_{E}^{n}$ must be taken from the world data. Generally, the contribution from the $G_{E}$ is small.) However, with the energies considered here, there is not enough kinematic lever arm for such separation. A procedure to obtain $G_{M}^{n}$ is to model $g_{a}$ (e.g. with a dipole form) using our accurate mapping and assume this form to extract $G_{M}^{n}$. The caveat of this procedure is that $G_{M}^{n}$ then depends on the form assumption. At higher beam energies $(\sim 1 \mathrm{GeV})$, a kinematic separation is possible and $G_{M}^{n}$ can be obtained model independently. However, $G_{E}^{n}$ is still out of reach because at these energies, the contribution from the electric form factors to the cross section becomes negligible.

\section{HIGHER ENERGY EXPERIMENT}

It is desirable to also perform the experiment at beam energies near a few $\mathrm{GeV}$ because it would provide $g_{A}\left(Q^{2}\right)$ in an unmeasured $Q^{2}$ domain and permits a model-independent separation of $g_{A}$ and $G_{M}^{n}$. Furthermore, the backward electrons detected in coincidence with neutrons can allow access to the almost unknown pseudo-scalar form factor, $g_{P}$, up to $Q^{2}$ of a few $\mathrm{GeV}^{2}$. Presently, only 3 data points exist at $Q^{2}<0.15 \mathrm{GeV}^{2}$ [11]. At $1 \mathrm{GeV}$, the weak/EM cross section ratio is larger by a factor $\sim 30$ but new experimental difficulties arise that actually make the experiment more difficult. An inelastic EM background with neutrons present in the final state appears above the pion production threshold. This EM background has a non-zero single spin asymmetry which can void the clean background subtraction scheme using a polarized beam. However, this EM asymmetry averages out when integrated over the polar angle. 
Hence, a detector setup (neutron and recoil detectors) symmetric around the beam line restores the subtraction scheme. Another difficulty is that the neutron detector should be placed at least $20 \mathrm{~m}$ away from the target to separate the relatively fast elastic neutrons from the photons. To keep a large solid angle we need a larger (more expensive) neutron detector. However, it also implies a better angular resolution and it minimizes the contribution of the target length to the TOF uncertainty. Nevertheless, the experiment is clearly challenging at high energy. It is thus necessary to gain experience from the low energy experiment before embarking on the higher energy one.

\section{CONCLUSION}

We discussed the feasibility of a pioneering weak charged current experiment. It allows to measure the axial-vector form factor $g_{A}\left(Q^{2}\right)$ with precision and accuracy typical of nucleon-electron scattering. It also allows the unique opportunity to access the free neutron magnetic form factor $G_{M}^{n}\left(Q^{2}\right)$. The experiment appears feasible without requiring any new technology. Within two months of running (60\% detection efficiency, 85\% beam polarization, signal/backgroud $\leq 1$ ) at a luminosity of $6 \times 10^{38} \mathrm{~cm}^{-2} \mathrm{~s}^{-1}$, it can provide a 14-points $Q^{2}$-mapping of $g_{A}$ from 0.009 to $0.039 \mathrm{GeV}^{2}$ with a sub-percent statistical uncertainty on each point. The systematic uncertainty is expected to be $4 \%$. From this mapping, if the dipole behavior of $g_{A}\left(Q^{2}\right)$ is confirmed, the axial mass can be extracted with negligible statistical uncertainty. This experiment would also be a stepping stone to more challenging higher energy experiments using charged current with electron beams. These would allow $g_{A}$ to be mapped in an unmeasured $Q^{2}$ range, to extract model-independently $G_{M}^{n}$ and to measure the elusive induced pseudoscalar form factor $g_{p}$. Such experiments at low and higher energy open new possibilities for nucleon structure study and searches beyond the standard model.

Acknowledgments The work in [5] was done in collaboration with D. Lhuillier. We thank D. Dutta for useful discussions. Jefferson Science Associates operates the Thomas Jefferson National Accelerator Facility under DOE contract DE-AC05-06OR23177.

[1] C. Alexandrou et al. Phys. Rev. D83 045010 (2011)

[2] V. Bérnard, L. Elouadrhiri, U-G. Meissner. J. Phys. G28 R1-R35 (2002)

[3] K2K Collaboration: R. Gran et al. Phys. Rev. D74 (2006) 052002

[4] MiniBooNE Collaboration: A. A. Aguilar-Arevalo et al. Phys. Rev. D81 092005, 2010

[5] A. Deur. JLab LOI 04-006 to PAC25. www.jlab.org/exp rog/proposals/04/LOI - 04 - 006.ps

[6] D. Dutta, these proceedings.

[7] T. W. Donnelly, E. L. Kronenberg and B. E. Norum (Research Program at CEBAF. 1985 summer study group); W-Y. Pauchy Hwang, ibid.

[8] D. S.Armstrong and R. D. McKeown, Ann. Rev. Nucl. Part. Sci. 62, 337 (2012), Y. Kolomensky, these proceedings.

[9] CLAS Collaboration: F. X. Girod et al. Phys. Rev. Lett. 100, 162002 (2008)

[10] PrimEx collaboration: I. Larin et al, Phys. Rev. Lett.106 162303 (2011)

[11] L. Ahrens et al. Phys. Rev. D35, 785 (1987); S. Choi et al. Phys. Rev. Let. 71, 3927 (1993) 